\title{
ON REGULAR NEIGHBORHOODS OF SPHERES
}

\author{
BY LAWRENCE S. HUSCH ${ }^{1}$
}

Communicated by J. Milnor, April 5, 1966

Consider the following two conjectures:

$C(n)$ : (The combinatorial Schoenflies conjecture.) A combinatorial $(n-1)$-sphere on a combinatorial $n$-sphere decomposes the latter into two combinatorial $n$-cells.

$D(n)$ : Let $W^{n}$ be an orientable combinatorial manifold without boundary and let $M^{n-1}$ be a closed orientable combinatorial manifold imbedded piecewise linearly in $W^{n}$. Let $U$ be a regular neighborhood of $M^{n-1}$ in $W^{n}$. Then there exists a piecewise linear homeomorphism $h: M^{n-1} \times[-1 ; 1] \rightarrow U$ such that

$$
\begin{aligned}
& h(x, 0)=x, \\
& h \text { is onto. }
\end{aligned}
$$

It is easily seen that $D(n)$ implies $C(n)$ for all $n \neq 4$ by using the Hauptvermutung for combinatorial cells and spheres [10]. In [8], Noguchi shows that $C(1), C(2), \cdots, C(n)$ imply $D(n+1)$. By using the fact that a compact component of the boundary of a combinatorial manifold is combinatorially collared [9], [11], it is easily shown that $C(n)$ implies $D(n+1)$. However it is possible to prove a weaker version of $D(n+1)$ without the use of $C(n)$ for the special case when $W, M$ are spheres.

Theorem. Let $\sum^{n}(n \neq 4)$ be a combinatorial sphere embedded piecewise linearly in the combinatorial sphere $S^{n+1}$. Let $U$ be a regular neighborhood of $\sum^{n}$ in $S^{n+1}$. Then there exists a piecewise linear homeomorphism $h: \sum^{n} \times[-1 ; 1] \rightarrow S^{n+1}$ such that $h\left(\sum^{n} \times[-1 ; 1]\right)=U$.

Proof. (For definitions of terms used see [11].) Since $C(i), i=1,2,3$, is valid [1], [6], it follows from the remarks above that the theorem is true for $n<4$. Suppose $n>4$.

Since $\sum^{n}$ is a deformation retract of $U$, the $i$ th integral homology groups of $\sum^{n}$ and $U$ are isomorphic for all $i$. It follows then from Alexander duality and the unicoherence of the sphere that the closure of $S^{n+1}-U, \mathrm{Cl}\left(S^{n+1}-U\right)$, is the union of two connected closed sets,

1 The contents of this paper form a part of the author's dissertation submitted as a partial requirement for the Ph.D degree at Florida State University under the direction of Professor James J. Andrews. Research was supported by a National Science Foundation Cooperative Graduate Fellowship. 
$D_{1}, D_{2}$ with a connected boundary $T_{1}, T_{2}$ respectively. Since $U$ is a combinatorial manifold, from [2], we have that each $D_{i}$ is a combinatorial manifold. Similarly, $S^{n+1}-\sum{ }^{n}=R_{1} \cup R_{2}$ where $D_{i} \subset R_{i}$ and $\mathrm{Cl} R_{1} \cap \mathrm{Cl} R_{2}=\sum{ }^{n}$. By either [3] or [7], $\mathrm{Cl} R_{1}$ and $\mathrm{Cl} R_{2}$ are topological $(n+1)$-cells.

We want to show that each $T_{i}$ is simply connected. Let $f: S^{1} \rightarrow T_{1}$ be a continuous map of the 1 -sphere into $T_{1}$. By the simplicial approximation theorem, we may assume $f$ is piecewise linear. Since $U$ is simply connected (for it is of the same homotopy type as $\sum n$ ), $f\left(S^{1}\right)$ bounds a disk $N$ in $U$. We may assume $N$ is polyhedral and in general position with respect to $\sum^{n}$. Then if $N \cap \sum^{n} \neq \varnothing, N \cap \sum^{n}$ is a finite collection of simple closed curves. Since $\sum^{n}$ is simply connected, we can suppose that $N$ lies in $U \cap \mathrm{Cl} R_{1}$; for by the usual alteration techniques, see, for example, [4], we can replace $N$ by a disk which is bounded by $f\left(S^{1}\right)$ and lies in $U \cap C l R_{1}$. By using the collar of the boundary of $\mathrm{Cl} R_{1}$, we can assume that $N \cap \sum^{n}=\varnothing$. Since $U-\sum{ }^{n}$ $=\left(T_{1} \cup T_{2}\right) \times[0,1)$, we can then push $N$ into $T_{1}$.

Since $D_{i} \cup U \searrow \mathrm{Cl} R_{i}, i=1,2$, it follows that each $D_{i} \cup U$ is contractible and hence from the fact that each $T_{i}$ is bicollared and from duality, each $D_{i}$ has homology groups of a point. Since each $T_{i}$ is simply connected it follows from a similar argument as above that each $D_{i}$ is simply-connected. From the Hurewicz Isomorphism Theorem, it follows that each $D_{i}$ is contractible. Hence from [10], we have that each $D_{i}$ is a combinatorial $(n+1)$-cell. From [2], each $\mathrm{Cl}\left(S^{n+1}-D_{i}\right)$ is a combinatorial $(n+1)$-cell. Then $U=\mathrm{Cl}\left(\mathrm{Cl}\left(S^{n+1}\right.\right.$ $\left.\left.-D_{1}\right)-D_{2}\right)$ is piecewise linear homeomorphic to $\sum n \times[-1 ; 1][11]$.

REMARKs. Attempts to prove the above theorem for manifolds not spheres by the techniques of Noguchi fail because of the missing dimension $n=4$. From [5], it follows that $T_{1} \times(0 ; 1)$ is topologically homeomorphic to $S^{n} \times(0 ; 1)$, but otherwise it is unknown to the author whether $T_{1}$ is a topological 4 -sphere in the case $n=4$.

\section{REFERENCES}

1. J. W. Alexander, On the subdivision of 3-space by a polyhedron, Proc. Nat. Acad. Sci. U.S.A. 10 (1924), 6-8. 320.

2. - The combinatorial theory of complexes, Ann. of Math. 31 (1930), 292-

3. M. Brown, Locally flat embeddings of topological manifolds, Ann. of Math. $\mathbf{7 5}$ (1962), 331-341.

4. O. G. Harrold, Jr. and E. E. Moise, Almost locally polyhedral spheres, Ann. of Math. 57 (1953), 575-578.

5. K. W. Kwun and F. Raymond, Mapping cylinder neighborhoods, Michigan Math. J. 10 (1963), 353-357. 
6. E. Luft, On the combinatorial Schoenflies conjecture, Proc. Amer. Math. Soc. 16 (1965), 1008-1011.

7. M. H. A. Newman, On the division of Euclidean $n$-space by topological $n-1$ spheres, Proc. Royal Soc. London 257 (1960), 1-12.

8. H. Noguchi, The thickening of combinatorial n-manifolds in $(n+1)$-space, Osaka Math. J. 12 (1960), 97-112.

9. R. Penrose, J. H. C. Whitehead, and E. C. Zeeman, Imbedding of manifolds in euclidean space, Ann. of Math. 73 (1961), 613-623.

10. S. Smale, Differentiable and combinatorial structures on manifolds, Ann. of Math. 74 (1961), 498-502.

11. E. C. Zeeman, Seminar on combinatorial topology, Inst. Hautes Études Sci. Publ. Math., Paris, 1963. (mimeographed notes)

Florida State University 